\title{
Supplementary Material: Nitrogen challenges and opportunities for agricultural and environmental science in India
}

\section{Participant list}

Table S1: List of the participants of the conference and workshop titled "Challenges and Opportunities for Agricultural Nitrogen Science in India" and their affiliations.

\begin{tabular}{|c|c|}
\hline Name & Institute \\
\hline Aakansha Malik & Guru Gobind Singh Indraprastha University \\
\hline Aakriti Wanchoo-Kohli & Rothamsted Research \\
\hline Adam Price & Aberdeen University \\
\hline Aditi Sharma & British High Commission \\
\hline Aimable Uwizeye & Food and Agriculture Organisation of United Nations \\
\hline Alison Bentley & NIAB \\
\hline Altaf Ahmad & Aligarh Muslim University \\
\hline Ambreen Asif & Aligarh Muslim University \\
\hline Amresh Chaudhary & Indian Agricultural Research Institute \\
\hline Anandan Annamalai & National Rice Research Institute \\
\hline Andrea Móring & University of Edinburgh \\
\hline Andrew Telford & RCUK India \\
\hline Aniket Kumar Singh & Indian Agricultural Research Institute \\
\hline Anil Kumar Saxena & $\begin{array}{l}\text { National Bureau of Agriculturally Important } \\
\text { Microorganisms }\end{array}$ \\
\hline Anirban Basu & University of Hyderabad \\
\hline Anita Kumawat & Indian Agricultural Research Institute \\
\hline Annapurna Kannepalli & Indian Agricultural Research Institute \\
\hline Arti Bhatia & Indian Agricultural Research Institute \\
\hline Bhumika Madan & Guru Gobind Singh Indraprastha University \\
\hline C.N. Neeraja & Indian Institute of Rice Research \\
\hline D.L.N. RAO & Indian Institute of Soil Science \\
\hline Dali Rani Nayak & University of Aberdeen \\
\hline Danteswari Chalasani & University of Hyderabad \\
\hline Dinesh Kumar & Indian Agricultural Research Institute \\
\hline Dinesh Kumar Jaiswal & Guru Gobind Singh Indraprastha University \\
\hline Edward Carnell & Centre for Ecology \& Hydrology \\
\hline Emma Wallington & NIAB \\
\hline Himanshu Pathak & National Rice Research Institute \\
\hline Howard Griffiths & University of Cambridge \\
\hline Irum Rizvi & Guru Gobind Singh Indraprastha University \\
\hline Jagdish Ladha & UC - Davis \\
\hline
\end{tabular}




\begin{tabular}{|c|c|}
\hline Jayesh Singh & Punjab Agricultural University \\
\hline Jo Smith & University of Aberdeen \\
\hline John Foulkes & University of Nottingham \\
\hline Julia Drewer & Centre for Ecology \& Hydrology \\
\hline Mahender Kumar Rapolu & Indian Institute of Rice Research \\
\hline Mangal Deep Tuti & Indian Institute of Rice Research \\
\hline Manoj Prasad & National Institute of Plant Genome Research \\
\hline Mark Sutton & Centre for Ecology \& Hydrology \\
\hline Mohd Irfan Naikoo & Aligarh Muslim University \\
\hline N. Raghuram & Guru Gobind Singh Indraprastha University \\
\hline Narendra Sharma & Guru Gobind Singh Indraprastha University \\
\hline Naresh Kumar Gattupalli & Maharaja Sayajirao University of Baroda \\
\hline Neha Bhardwaj & Guru Gobind Singh Indraprastha University \\
\hline Niveta Jain & Indian Agricultural Research Institute \\
\hline P.B. Kirti & University of Hyderabad \\
\hline Pallavolu Maheswara Reddy & The Energy and Resources Institute \\
\hline Parveen Chhuneja & Punjab Agricultural University \\
\hline Peter Shewry & Rothamsted Research \\
\hline Philip Poole & University of Oxford \\
\hline Pooja Pawar & Shivaji University \\
\hline Pranab Kumar Mandal & National Research Centre on Plant Biotechnology \\
\hline Ramakrishnan Balasubramanian & Indian Agricultural Research Institute \\
\hline Ray Dixon & John Innes Centre \\
\hline Renu Pandey & Indian Agricultural Research Institute \\
\hline Renu Singh & Indian Agricultural Research Institute \\
\hline Rita Sharma & Newton Fund India \\
\hline Roger Sylvester-Bradley & ADAS \\
\hline Sahaj Kaur & TERI University \\
\hline Sanjoy Bandyopadhyay & Indian Agricultural Research Institute \\
\hline Santosh Mohanty & Indian Institute of Soil Science \\
\hline Santosh P. Deshpande & ICRISAT \\
\hline Saran Sohi & University of Edinburgh \\
\hline Sarma Pvsrn & University of Hyderabad \\
\hline Satinder Kaur Kaur & Punjab Agricultural University \\
\hline Seema Sepat & Indian Agricultural Research Institute \\
\hline Shanti Devi Bamboriya & Indian Agricultural Research Institute \\
\hline Shweta Mehrotra & Indian Agricultural Research Institute \\
\hline Stephanie Smith & Cambridge University \\
\hline Stéphanie Swarbreck & University of Cambridge \\
\hline Subodh Kumar Sinha & National Research Centre on Plant Biotechnology \\
\hline Subrahmanyam Desiraju & Indian Institute of Rice Research \\
\hline
\end{tabular}




\begin{tabular}{|l|l|}
\hline Sunila Hooda & Guru Gobind Singh Indraprastha University \\
\hline Surekha Kuchi & Indian Institute of Rice Research \\
\hline Surendra Singh Chauhan & Indian Agricultural Research Institute \\
\hline Tapan Adhya & KIIT University \\
\hline Till K Pellny & Rothamsted Research \\
\hline Tina Barsby & NIAB \\
\hline Tirthankar Bandyopadhyay & National Institute of Plant Genome Research \\
\hline Varinderpal Singh & Punjab Agricultural University \\
\hline Vasudev Kumanduri & NIAB \\
\hline Venkatesh Karnam & Indian Institute of Wheat and Barley Resaerch \\
\hline Vikas Kumar Mandal & Guru Gobind Singh Indraprastha University \\
\hline Vinod Goyal & CCS Haryana Agriculture University \\
\hline Vinoy Ramachandran & University of Oxford \\
\hline
\end{tabular}

